\title{
Introduction of an online discussion forum and electronic communication practice in a tertiary-level Anaesthesia Department
}

\author{
Brenton Sanderson, Jeremy Field \\ Department of Anaesthesia and Perioperative Medicine, Westmead Hospital, Australia
}

Received: September 13, 2018

DOI: $10.5430 /$ jha.v7n6p24
Accepted: November 4, $2018 \quad$ Online Published: November 9, 2018

URL: https://doi.org/10.5430/jha.v7n6p24

\begin{abstract}
Objective: Electronic communication mediums provide an opportunity for clinicians to enhance communication, collaboration, and sharing of clinical experience, especially via mobile devices. In 2016, the authors implemented a private online discussion forum in a tertiary-level anaesthesia department to improve communication and collaboration amongst members. The objective of this survey was to assess if these aims were met, to determine the degree of communication medium duplication incurred by its introduction, and to assess departmental communication practices more generally.

Methods: A qualitative anonymous online survey was conducted 18 months following the introduction of the online discussion forum, over a two-month period. All 120 medical staff were invited via forum message and hard-copy invitation and responses were stratified by training status.

Results: Forty-seven responses were collected (39\% response rate), comprising 31 anaesthesia specialists and 16 anaesthesia trainees. Sixty-one percent of respondents reported that discussion had improved following the introduction of the online discussion forum, with no significant difference between specialists and trainees. Despite this, 57\% overall maintained a preference for email discussion.

Conclusions: This study demonstrated that the introduction of an online discussion forum resulted in a perceived improvement in overall departmental communication. However, it is important that workplaces considering implementation of a similar communication medium determine their employees' cultural perspectives on technology, established communication preferences and aims of implementation to ensure success.
\end{abstract}

Key Words: Online discussion, Communication, Mobile application, Collaboration

\section{INTRODUCTION}

Communication within hospital departments has transformed dramatically over the last few decades, from pieces of paper pinned on notice boards to instantaneous mobile interaction using myriad applications and technologies. Although ubiquitous, the sheer volume of email makes discussions difficult to track and follow in contemporary workplaces. ${ }^{[1]}$ Use of email also makes it difficult to maintain a "corporate memory" of previous dialogue on a subject, especially as new staff join the department. ${ }^{[2]}$ There are now countless online communication platforms purporting to solve these problems, ranging from general purpose to healthcarespecific. ${ }^{[3-5]}$ However, the range of solutions and widespread

*Correspondence: Brenton Sanderson; Email: brenton.sanderson@health.nsw.gov.au; Address: Department of Anaesthesia and Perioperative Medicine, Westmead Hospital, Australia. 
use of similar platforms and social media for non-work related communication make selecting the appropriate platform for an entire workplace challenging. ${ }^{[6]}$

Change in departmental communication practice is not just a technological issue, but also relates to culture, perspectives on innovation, and change in both the organisation and the individual. ${ }^{[7]}$ Funding is also required, and may be in short supply, especially in a public healthcare system. Successful change in healthcare communication, like change in clinical practice, requires high quality evidence, clear context and comprehensive facilitation. ${ }^{[8]}$
In October 2016, the authors configured and deployed a new private online discussion forum for use within the Department of Anaesthesia and Perioperative Medicine, using the free, open-source platform phpBB (phpBB Limited, Marlow, Bucks, UK) with mobile access facilitated via Tapatalk (Tapatalk, Santa Monica, Ca, USA). The forum was accessible via the web, shown in Figure 1, and via the custom mobile application, shown in Figure 2. The forum has been implemented in parallel to existing communication media, mainly email, and participation is not compulsory.

\section{Westmead Anaesthesia}

$$
\text { n Board index }
$$

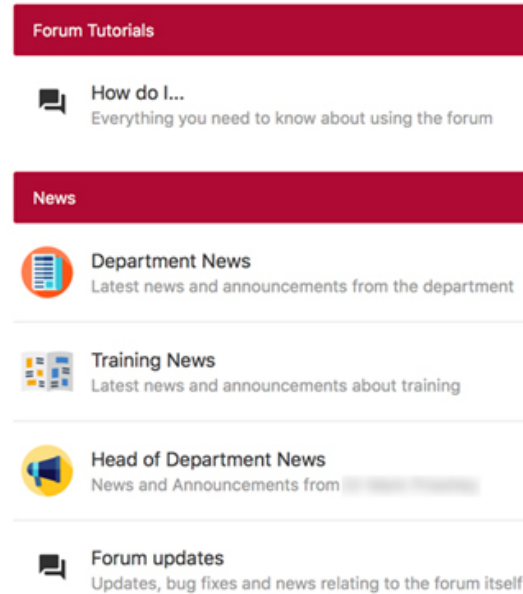

Updates, bug fixes and news relating to the forum itself
$24 \quad 30 \quad$ Congratulations on a brillian...

$\begin{array}{cccc}24 & 30 & \text { Congratulations on a brillian.... } \\ \text { Topics } & \text { Posts } & \text { by }\end{array}$

$\begin{array}{ccl}2 & 2 & \text { New forum category - Recommen.. } \\ \text { Topics } & \text { Posts } & \text { by }\end{array}$
$14 \quad 14 \quad$ How do I fix the issue of una... Topics Posts by 2 months ago

Search.

Mark forums read

(2) Who is online

Registered users:

minutes

wh. Statistic

Total topics

Total posts

Total members

Our newest member

$\begin{array}{ccc}17 & 18 & \text { JournalClub Allocations }- \text { Upd... } \\ \text { Topics } & \text { Posts } & \text { by }\end{array}$

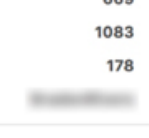

\section{Allocations}

Consultants

Monthly allocation

Registrar allocations, terms and rosters
(1)

\section{9


forum and general online communication practices within our department.

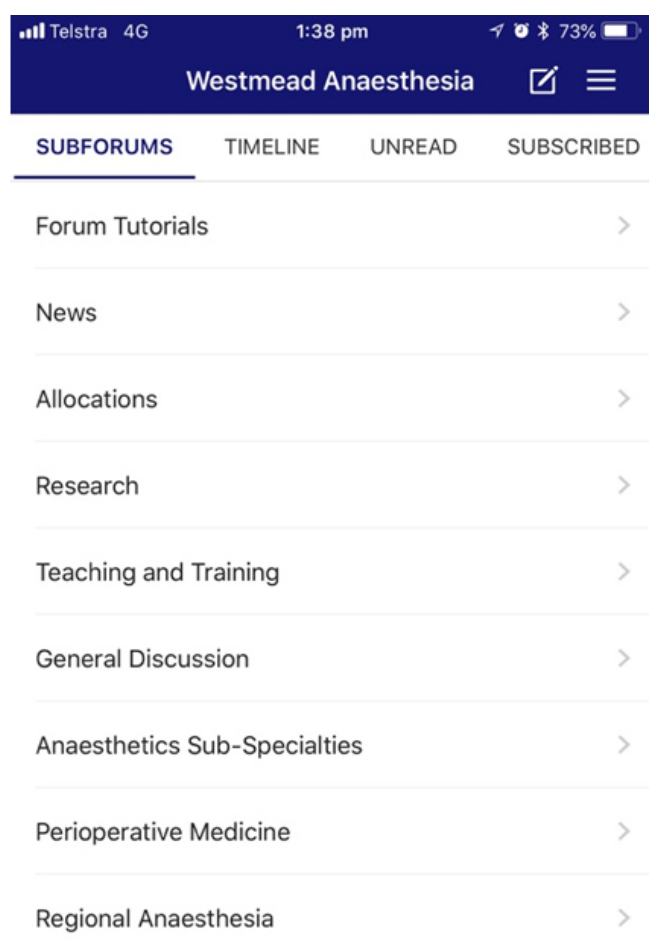

Figure 2. Westmead Anaesthesia Forum (via Tapatalk mobile application)

\section{Methods}

\subsection{Study design and participants}

We conducted a qualitative anonymous online survey to evaluate the efficacy of the online discussion forum. The study was conducted over a two-month period from March 2018. All anaesthesia specialists and trainees within the Department of Anaesthesia and Perioperative Medicine at Westmead Hospital, Sydney, Australia were invited to participate.

\subsection{Survey}

The survey was structured to evaluate electronic communication practices and the impact of the online discussion forum. The survey was organised into two sections. The first section related to user preference among electronic group communication mediums and usage of online discussion forums in general, prior to the implementation of our own forum. The second section related to usage of the new forum and evaluation of online communication practice following its introduction. The survey consisted of 30 questions, using the web-based survey tool, SurveyMonkey (Portland, OR, USA). Twenty questions were multiple choices, four questions were dichotomous and six questions were open ended optional comment fields. A summary of themes addressed by the survey is shown in Table 1.

\subsection{Data collection}

All 120 anaesthesia medical staff (88 specialists and 32 trainees) were invited via both an advertisement on the forum and paper-based invitations within the department. Respondents were requested to provide their level of training/years of specialist practice as appropriate. This formed the basis of comparative group analysis. No other identifying information was requested.

Table 1. Summary of themes addressed by online survey

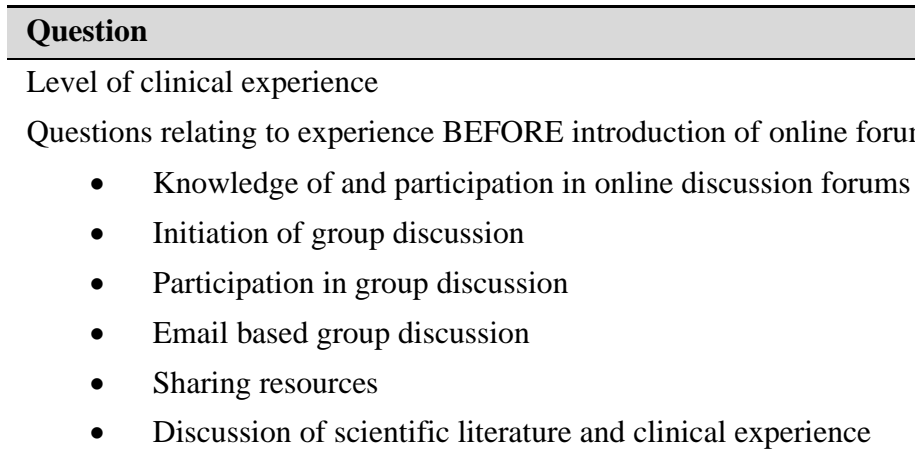

Questions relating to experience AFTER introduction of online forum

- Forum access frequency and device/s used

- Forum ease of use and likeliness to use for discussion and sharing of resources

- Comparison to email and change in communication practice

- Change in discussion of scientific literature and clinical experience

- Change in clinical practice and ability to maintain knowledge of current literature 


\subsection{Analysis}

Survey responses were analysed using SPSS (version 24, IBM Corp., Somers, NY, USA). All questions other than open ended questions were analysed with cross tabulation to identify proportions and compare responses of anaesthesia trainees versus anaesthesia specialists. Comment based questions were summarised by theme. Responses to multiple choice questions were analysed using the chi-square test and Fisher's exact test when expected cell count was $<5$, with statistical significance considered as two tailed and $p<.05$.

\section{RESULTS}

One hundred and twenty staff were invited to participate: 47 completed the survey, giving a $39 \%$ response rate. Sixteen out of $32(50 \%)$ anaesthesia trainees and 31 out of 88 (35\%) anaesthesia specialists responded to the survey. Prior to the introduction of the forum, $89 \%$ of respondents had knowledge of online discussion forums and $51 \%$ had used one.

\subsection{Group discussion prior to forum}

Prior to the introduction of the forum, respondents overall preferred to initiate group discussion using email (53\%), followed by in-person (30\%) and messaging applications $(15 \%)$, with specialists and trainees having equal preference for email and messaging applications. For those respondents who preferred email, $80 \%$ were satisfied or very satisfied with this medium, with no difference between trainees and specialists $(p=.56)$.

When respondents were invited to participate in a group discussion the preferred communication medium overall was messaging application (34\%) followed by email (26\%). For specialists, email was the preferred medium $(32 \%)$ whereas trainees preferred messaging applications (63\%) over other group communication media.

\subsection{Group email discussion prior to forum}

Forty five percent of respondents reported they were likely or very likely to start a group discussion via email, specialists $(58 \%)$ more so than trainees $(19 \%)$. Training status was associated with likeliness to start a group discussion via email $(p=.04)$.

When respondents were invited to an email group discussion, $64 \%$ (77\% of whom were specialists) were likely or very likely to reply. Trainees, conversely, were neither likely nor unlikely to reply, however there was no statistically significant relationship between likelihood of response and training status $(p=.1)$. Comments relating to likeliness to respond when invited to a group email discussion mainly related to feeling strongly about the issue being discussed or feeling comfortable with the discussion participants.

\subsection{Sharing resources and experience prior to forum}

The majority of respondents preferred to use email for sharing resources $(79 \%)$. This preference was consistent for both specialists and trainees $(p=.39)$. Amongst those who preferred email for sharing resources, $84 \%$ were satisfied or very satisfied with this medium with $81 \%$ of respondents being satisfied or very satisfied with their chosen medium overall. The main reasons given for satisfaction with email related to a perception that email was easy to use and allowed control over specific recipients.

Forty percent of respondents overall reported reading a scientific journal article at least weekly. Specialists reported reading a journal article most commonly on a monthly basis $(42 \%)$ whereas trainees most frequently reported reading a journal article on at least a weekly basis $(44 \%)(p=.58)$.

Discussion of clinical experience with a colleague was reported to occur most frequently on a weekly basis by $49 \%$ of respondents overall. This pattern was reflected by both specialists and trainees $(p=.84)$.

\subsection{Access to online discussion forum}

Respondents accessed the forum predominantly on a weekly basis $(48 \%)$ with no difference between specialists and trainees $(p=.79)$. Seventy-six percent of respondents reported not accessing the forum as frequently as they would like, which included $84 \%$ of specialists and $60 \%$ of trainees $(p=.08)$.

The most frequent reason given for not accessing the forum as much as desired was a perceived lack of time in $40 \%$ of overall respondents. Other reasons included inability to login to the forum, competition with other electronic communication mediums and speed of access to the forum.

The forum was most commonly accessed using a mobile phone ( $80 \%$ of respondents), of which $76 \%$ ran iOS and $24 \%$ Android. Both specialists $(77 \%)$ and trainees $(93 \%)$ predominantly accessed the forum using a mobile phone. Learning how to use the forum was perceived by $67 \%$ of respondents to be easy or very easy, with a similar result in both the specialist and trainee groups $(p=.6)$.

\subsection{Sharing resources and experience following intro- duction of the forum}

Thirty-seven percent of respondents reported they were likely or very likely to use the forum for discussion or sharing of resources, comprising $36 \%$ of specialists and $40 \%$ of trainees. Thirty percent of respondents were equivocal. Comments relating to this preference from the perspective of specialists 
related to an unfamiliarity with the medium and a lack of guaranteed audience whereas trainees reported a perceived lack of engagement from colleagues and availability of more convenient options for sharing information. In comparison to the forum, $57 \%$ of overall respondents somewhat or definitely prefer email for discussion and sharing of resources. This preference was similar from both specialists (61\%) and trainees (47\%). Reasons given for satisfaction with email mainly related to its perceived widespread use and the ability to engage with specific individuals.

Despite this, $61 \%$ of overall respondents reported that sharing of resources and discussion had somewhat or definitely improved with the introduction of the forum (53\% of trainees and $65 \%$ of specialists, $p=.47$ ).

\subsection{Review of scientific literature following introduction of the forum}

Forty-six percent of respondents overall reported reading scientific literature on the forum on a monthly or more frequent basis, with similar frequencies among specialists and trainees. When asked about scientific literature found elsewhere, $50 \%$ of respondents overall reported reading at least weekly and $85 \%$ reported reading at least monthly. Again, these frequencies were similar in both specialist and trainee groups.

Forty-eight percent of overall respondents reported the forum to have somewhat or definitely improved their ability to maintain their knowledge of current literature, comprising $45 \%$ of specialists and $53 \%$ of trainees. When asked if information found on the forum had changed any aspect of their clinical practice, $26 \%$ of specialist and $13 \%$ of trainee respondents replied in the affirmative.

\subsection{Overall comments on forum}

Overall comments from trainee respondents included suggestion of a weekly email notification for new forum content, issues with lack of perceived participation and corresponding user engagement and the difficulty of choosing amongst multiple available electronic communication platforms.

Overall comments from specialist respondents related to the lack of participation from colleagues and therefore reluctance to participate or initiate discussion without a guaranteed audience in comparison with email. It was suggested that with greater departmental engagement, the utility of the forum as a communication medium would also improve.

\subsection{Limitations}

Our anonymous, voluntary, partly retrospective online survey is vulnerable to both recall bias and participation bias. Studying the use of an online forum using an online survey is likely to bias against members of the study population who may be less able or interested to participate in online work-related activities.

Future studies could be enhanced by the additional analysis of content and metadata both from the forum, and from competing group communication media such as email, providing that user consent (both of participants and non-participants) could be acceptably addressed.

The variability of electronic communication practice across different organisations, individuals and society as a whole, may render the findings of this study inapplicable to other organisations or departments.

Our participation rate of $39 \%$ is less than the established average response rate for organisational research of $52.7 \%$; this may limit the extent to which the study represents the opinions of the department as a whole. ${ }^{[15]}$

\section{Discussion}

A private online discussion forum was introduced to a tertiary anaesthesia department to promote discussion and sharing of resources. It was implemented in parallel to existing communication mediums, mainly email, without compulsory involvement. This survey, conducted approximately 18 months following implementation, demonstrates that overall discussion has improved despite perceived limited engagement and a preference for existing mediums, particularly email. Implementation of any new electronic communication platform, similarly to any new Healthcare Information System in this context, is not without challenges, as we have demonstrated. ${ }^{[16]}$ The results of this survey represent a minority (39\%) of anaesthesia providers in the department. The experience of non-respondents may differ and should be explored in subsequent studies with the possibility of promoting engagement through addressing concerns.

The phpBB platform was chosen for its maturity, free and open-source licence and customisation capabilities (including community-contributed modules). Tapatalk was used to enhance mobile phone access via a customised, commercially licenced application. Implementation did not require a significant financial investment and made use of an existing department internet server.

Online communication mediums present an opportunity to extend the accessibility and enrich the efficacy of learning. ${ }^{17]}$ This benefit to the learning process not only applies to specialty trainees but also forms another avenue for established specialists to maintain current and attain new knowledge and experience. Our forum was used to share teaching material, and to discuss current scientific literature and the application 
of this knowledge, interpreted through clinical experience. The forum also facilitated our teaching program for trainees that were rotating to multiple hospitals throughout their training.

\section{Conclusions}

This survey reflects our experience of implementing an online discussion forum in a tertiary level anaesthesia department, showing that it has improved perceived departmental communication, although still competes with email and instant messaging for users' attention. The forum has also allowed all members to benefit from our collective departmental clin- ical experience and discussion. Based on our experience of the phpBB forum platform, we would recommend a different platform that is capable of sharing resources directly from mobile applications and easily creating discussion groups with targeted users. It is important that departments and organisations considering implementation of a similar communication medium determine their employees' perspectives on technology, established communication preferences, and aims of implementation to ensure success.

\section{CONFlicts of InTEREST Disclosure}

The authors declare they have no conflicts of interest.

\section{REFERENCES}

[1] Grevet C, Choi D, Kumar D, et al. Overload is overloaded: email in the age of Gmail. Proceedings of the SIGCHI Conference on Human Factors in Computing Systems. Toronto, Ontario, Canada: ACM; 2014. 793-802 p.

[2] Beckett RC. A characterisation of corporate memory as a knowledge system. Journal of Knowledge Management. 2000; 4(4): 311-9. https : //doi.org/10.1108/13673270010379867

[3] Vocera Communications Inc. Vocera. San Jose (USA). [cited 2018 Aug 08]. Available from: https : //www. vocera.com/

[4] Eko Communications Inc. Bring your team together with Ekoapp. Bangkok (Thailand). [cited 2018 Aug 09]. Available from: https : //www. ekoapp.com/

[5] Slack Technologies Inc. Where work happens I Slack. San Francisco (USA). [cited 2018 July 28]. Available from: https : //slack . com/

[6] Australian Communications and Media Authority. Mobile apps Emerging issues in media and communications Occasional paper 1. Melbourne (Australia); 2013.

[7] Büschgens T, Bausch A, Balkin DB. Organizational Culture and Innovation: A Meta-Analytic Review. Journal of Product Innovation Management. 2013; 30(4): 763-81. https ://doi .org/10.1111/ jpim. 12021

[8] Rycroft-Malone J, Kitson A, Harvey G, et al. Ingredients for change: revisiting a conceptual framework. Qual Saf Health Care. 2002; 11: 174-80. https://doi.org/10.1136/qhc.11.2.174

[9] Chari A, Gane SB. Instant messaging applications in healthcare: are we harnessing their potential? BMJ Innovations. 2018; 4(1): 5-8. https://doi.org/10.1136/bmjinnov-2017-000197
[10] Gould G, Nilforooshan R. WhatsApp Doc? BMJ innovations. 2016; 2: 109-10. https://doi.org/10.1136/bmjinnov-2016-000 116

[11] Thomas K. Wanted: a WhatsApp alternative for clinicians. BMJ: British Medical Journal (Online). 2018; 360. https : //doi .org/ 10. $1136 / \mathrm{bmj} . \mathrm{k} 622$

[12] Bones E, Hasvold P, Henriksen E, et al. Risk analysis of information security in a mobile instant messaging and presence system for healthcare. Int J Med Inform. 2007; 76(9): 677-87. https : //doi.org/10.1016/j.ijmedinf.2006.06.002

[13] Lluch M. Healthcare professionals' organisational barriers to health information technologies-a literature review. Int J Med Inform. 2011; 80(12): 849-62. https://doi.org/10.1016/j.ijmedinf. 201 1.09 .005

[14] Karsh BT. Beyond usability: designing effective technology implementation systems to promote patient safety. Quality and Safety in Health Care. 2004; 13(5): 388-94. https://doi.org/10.1136/ qshc. 2004.010322

[15] Baruch Y, Holtom B. Survey response rate levels and trends in organizational research. Human Relations. 2008; 61(8): 1139-1160. https://doi.org/10.1177/0018726708094863

[16] Cresswell K, Sheikh A. Organizational issues in the implementation and adoption of health information technology innovations: An interpretative review. International Journal of Medical Informatics. 2013; 82(5): e73-e86. PMid: 23146626. https://doi.org/10.1016/j ijmedinf .2012.10.007

[17] Balaji M, Chakrabarti D. Student Interactions in Online Discussion Forum: Empirical Research from 'Media Richness Theory' Perspective. Journal of Interactive Online Learning. 2010; 9(1): 1-22. 